\title{
A TCR-switchable cell death pathway in T-ALL
}

\section{Christine Tran Quang, Benedetta Zaniboni, Jacques Ghysdael}

The cell surface receptor for antigen in mature $\mathrm{B}$ (BCR) and T lymphocytes (TCR) is central to the adaptative immune response. Structurally, these receptors entails the association between clonotypic antigen binding chains (TCR $\alpha$ and TCR $\beta$ for the TCR), coupled to signaling chains (CD $3 \varepsilon, \gamma, \delta$ and the $\zeta$ chains for the TCR). Emergence of many non-Hodgkin B cell lymphomas subtypes is commonly associated with antigenic BCR activation, activating mutations in BCR signaling chains and downstream adapters/effectors. Likewise, progression of certain T cell lymphomas is associated with gain-of function alterations in TCR signaling components. For example, Sezary syndrome cutaneous T cell lymphoma shows upregulation of the TCR LAT adaptor in most cases and frequent activating mutations in the adaptor CARD11 and in phospholipase $\mathrm{C} \gamma 1$ [1].

$\mathrm{T}$ cell acute lymphoblastic leukemia (T-ALL) originates from the transformation of $\mathrm{T}$ cell progenitors, resulting in the accumulation of lymphoblasts arrested at specific stages of differentiation. T-ALL are classified into molecular subtypes characterized by abnormal expression of specific transcription factors (e.g. TAL1, LMO1/2, TLX1/3), involved in differentiation blockade. A number of additional genetic alterations are found across these subtypes, including activating mutations in NOTCH1 or the JAK/STAT pathway and inactivating mutations in several tumor suppressor genes [2]. TCR-expressing mature T-ALL represent about $50 \%$ of pediatric cases and $20 \%$ of adult cases. Whether TCR signaling contributes to leukemogenesis is unclear. Information gathered from T-ALL mouse models indicates that while signaling through the pre-TCR impinges upon leukemogenesis, the presence of a functional TCR is not critical [3-5]. TCR signaling is involved in a major developmental checkpoint during normal $\mathrm{T}$ cell development in the thymus. Thymocytes bearing a high affinity TCR for self-peptide/MHC complexes are deleted (negative selection) while those with a low affinity TCR survive and further differentiate into mature $\mathrm{T}$ cells (positive selection). We observed that co-expression of the TELJAK2 oncogene with a transgene encoding TCR-HY, which induces negative selection only in male mice, specifically compromised leukemia onset in males [4]. Importantly, in our new study [6], when leukemias obtained from females were transplanted in either male or female secondary recipients, only females succumbed to T-ALL. This indicated that the strong/sustained TCR activation associated with the negatively selecting TCRHY severely impaired leukemia maintenance. Consistent with this, stimulation of a TCR-negative cell line engineered to express the TCR-HY transgene by the DBY cognate antigen resulted in dose-dependent cell death [6]. Modern multi-agent chemotherapy has considerably improved T-ALL outcome. However, about $15 \%$ pediatric and $40 \%$ adult patients relapse and overall survival is still below $25 \%$, calling the search for alternative therapeutic approaches. As specific anti-TCR/CD3 antibodies can induce signaling, we investigated an immunotherapeutical approach using anti-CD3 $\varepsilon$ antibodies in T-ALL. In vitro treatment with anti-CD3 $\varepsilon$ specifically induced TCR signaling followed by apoptosis in 24/24 TCR-positive diagnostic T-ALL cases while sparing TCR-negative cases. Most importantly, in vivo expansion of 6/6 TCR-positive xenografts belonging to different T-ALL molecular subtypes was severely impaired by anti-CD $3 \varepsilon$ OKT3 $\mathrm{mAb}$ treatment, an anti-leukemic effect that translated into improved survival. OKT3 anti-leukemic effects can result from induction of a cell-intrinsic cell death program and/or antibody-dependent cell cytotoxicity (ADCC)-type responses. The fact that LAT expression knockdown in T-ALL strongly impaired the anti-leukemic response to OKT3 shows that, at least in NSG mice, OKT3-induced TCR signaling rather than ADCC-type responses is responsible for the anti-CD3 anti-leukemic effects [6]. Thus a latent cell death program, switchable by anti-CD3e treatment, can be induced in T-ALL, which dominates the many distinct oncogenic pathways active in different tumors (Figure 1). We identified the transcriptional program associated with anti-CD3 treatment in T-ALL in vitro [6] and in vivo (our unpubl. obs.) and found it to resemble that of thymocyte negative selection, but to be distinct from that resulting from inactivation of T-ALL oncogenes [6,7]. Characterization of critical molecular effectors of this cell death program is ongoing and will allow identifying either synthetic lethal partners of antiCD3 treatment or ways to bypass anti-CD3 itself to further improve the therapeutic potential of this pathway. This is important since our results show that the presence of TCRnegative subclones in otherwise TCR-positive T-ALL xenografts results in leukemia recurrence from OKT3mediated therapy [6].

The selection of acquired mutations during T-ALL progression is associated with clonal evolution, resulting in coexistence at diagnosis of related clones 
endowed with distinct leukemogenic potential. Whether anti-CD3 treatment can impair the leukemia initiating (stem) potential of TCR-positive T-ALL remains to be investigated.

OKT3 has been used in the clinics since 1986 to treat allograft rejection. Its side effects in humans include strong immunogenicity and a cytokine-release (flu-like) syndrome. Since then, several humanized anti-CD3 $\varepsilon$ $\mathrm{mAb}$ further mutated in their Fc domain to impair FcR recognition have been developed [8]. Pre-clinical testing of these $\mathrm{mAb}$ for their anti-leukemic activity is ongoing in T-ALL xenografts. Irrespective of the fact that anti-CD3based therapeutic approaches will or not prove useful in the clinic, our work in collaboration with the Asnafi laboratory [6] uncovered a conserved and switchable cell death pathway in T-ALL. Further dissection of this pathway to identify its intracellular effectors will provide alternatives to TCR-directed therapies that, in addition, might turn out to be relevant also to the treatment of TCRnegative T-ALL.

\section{CONFLICTS OF INTEREST}

The authors declare no conflicts of interest. Jacques Ghysdael: Institut Curie, PSL Research University, CNRS UMR 3348, F-91405, Orsay, France; Université Paris Sud, Université Paris-Saclay, CNRS UMR 3348, F-91405 Orsay, France
Correspondence: Jacques Ghysdael, email jacques.ghysdael@curie.fr

Keywords: T-ALL, TCR signaling, cell death, negative selection, targeted therapy

Received: March 01, 2017

Published: March 31, 2017

\section{REFERENCES}

1. Wang L, et al. Nat Gen. 2016; 47:1426-1434.

2. Belver L, et al. Nat Rev Cancer. 2016; 16:494-507.

3. Campese AF, et al. Blood. 2006; 108:305-310.

4. Dos Santo, et al. Blood. 2007; 109: 3972-81.

5. Tremblay M, et al. Genes and Dev. 2010; 24:1093-1105.

6. Trinquand, et al. Cancer Discovery. 2016; 6: 972-85.

7. Dadi S, et al. Cancer Cell 2012; 21: 563-76.

8. Kuhn C, et al. Immunotherapy 2016; 8: 889-906.

Copyright: Tran Quang et al. This is an open-access article distributed under the terms of the Creative Commons Attribution License (CC-BY), which permits unrestricted use, distribution, and reproduction in any medium, provided the original author and source are credited.

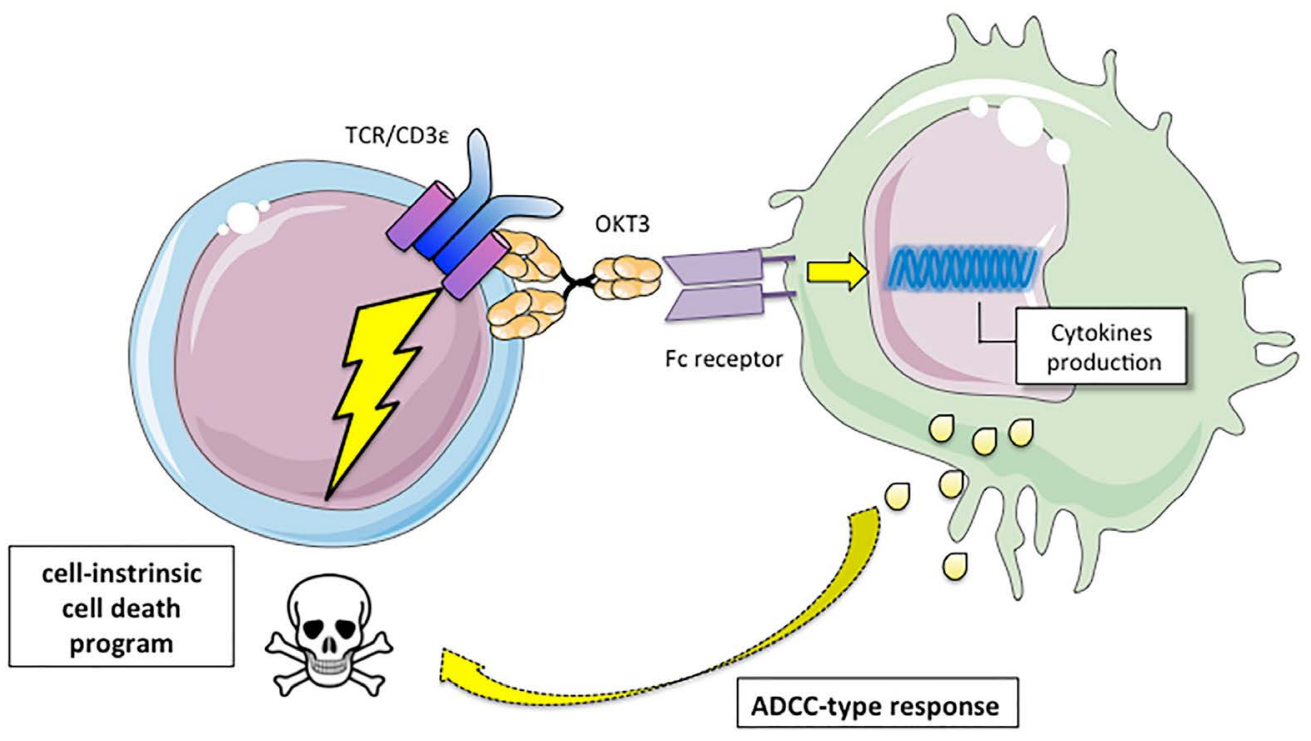

Figure 1: In T-ALL an intrinsic, TCR-induced cell death pathway activates leukemic cell apoptosis. 